05

\title{
Пластическая деформация [001]-монокристаллов никеля. Моделирование и эксперимент
}

\author{
() Е.А. Алфёрова, ${ }^{1}$ Е.В. Фомин ${ }^{2}$ \\ ${ }^{1}$ Национальный исследовательский Томский политехнический университет, \\ 634050 Томск, Россия \\ 2 Челябинский государственный университет, \\ 454001 Челябинск, Россия \\ e-mail: katerina525@mail.ru, fomin33312@gmail.com
}

(Поступило в Редакцию 7 марта 2018 г.)

С применением математического моделирования и натурных экспериментов рассмотрены процессы пластической деформации на примере [001]-монокристаллов никеля. Установлено, что способность кристалла к самоорганизации пластической деформации существует на всех масштабных уровнях, но наиболее сильно проявляется на микроуровне. Установлены механизмы самоорганизации пластической деформации на микро- и мезоуровне. В первом случае это согласованное зарождение и аннигиляция дислокаций, а во втором - коррелированный сдвиг в параллельных плоскостях скольжения. Благодаря указанным процессам распределение компонент деформации по грани носит квазипериодический характер, что способствует сохранению целостности кристалла при нагружении.

DOI: 10.21883/JTF.2019.01.46971.107-18

\section{Введение}

Пластическая деформация твердых тел реализуется за счет дефектов различной природы и протекает на различных масштабных уровнях. На сегодняшний день в руках исследователей сосредоточены различные инструменты для изучения природы пластической деформации. Это и эксперимент с последующим анализом полученных результатов и математическое моделирование соответствующих процессов.

В экспериментальных работах по изучению пластической деформации ГЦК-монокристаллов были установлены закономерности развития деформационного рельефа, предложены различные виды классификаций элементов рельефа, рассмотрены вопросы фрагментации, неоднородности и локализации деформации при различных условиях нагружения [1-5]. Был выявлен ряд закономерностей и механизмов пластической деформации, показан многоуровневый характер деформации и установлены черты самоорганизации [6-11].

В работах по моделированию [12-15] рассматривается формирование различного деформационного рельефа при образовании разного типа дислокационных структур, моделируется поведение дислокационного ансамбля, исследуются характеристические движения дислокаций под действием сдвиговых напряжений, исследуется взаимосвязь между атомистическим и макроуровнем. Кроме поведения дислокационных структур с помощью молекулярной динамики (МД) рассматриваются также напряженное состояние и изменение атомной структуры, например, [16], где исследовался отклик монокристалла под ударным воздействием - деформация образца осуществлялась за счет образования дефекта упаковки. В другой работе [17] с помощью МД авторы изучали фазовые превращения (ОЦК в ГПУ, ОЦК в ГЦК) кристаллической решетки при пластической деформации металлов с ОЦК-структурой. При моделировании всегда интересно провести сопоставление полученных данных с натурным экспериментом.

Таким образом, совместное применение этих двух подходов позволяет всесторонне изучить закономерности пластической деформации и выявить ее закономерности.

Целью настоящей работы является выявление физических закономерностей процесса пластической деформации и ее самоорганизации на различных масштабных уровнях с применением теоретических и экспериментальных методов.

\section{1. Материал и методика}

В качестве объекта исследования был выбран монокристаллический никель (чистота 99.99\%) с ориентацией оси сжатия [001] и боковыми гранями $\{110\}$. Деформировали сжатием образцы размером $3 \times 3 \times 6 \mathrm{~mm}$ на испытательной машине InstronElektroPuls E10000, скорость $1.4 \cdot 10^{-3} \mathrm{~s}^{-1}$. Картину деформационного рельефа исследовали на оптическом микроскопе Leica DM 2500 Р и конфокальном лазерном сканирующем микроскопе Olympus LEXT OLS4100. Размер сканированного участка в каждом отдельном случае составлял $0.066 \mathrm{~mm}^{2}$ $(0.256 \times 0.256 \mathrm{~mm})$. Разрешение по глубине $0.06 \mu \mathrm{m}$.

Для определения величины локальной деформации был использован метод делительных сеток. Угольную сетку с базой $100 \mu \mathrm{m}$ на недеформированной полированной поверхности образца получали напылением через медную сетку в вакуумном посту. Для уменьшения погрешности, связанной с отклонением размеров ячеек 
медной сетки, их измерение проводили до и после деформации. Определение величин компонент главных деформаций $e_{x}, e_{y}, e_{z}$ осуществляли по методике, изложенной в работе [18].

В качестве объекта моделирования использовали образец размером $7.04 \times 7.04 \times 21.12 \mathrm{~nm}$. По оси $X, Y$ (боковые грани кристалла $\{110\}$ ) применялись периодические граничные условия, а по $Z$ (ось сжатия [001]) непериодические. Расчеты проводились в программном пакете LAMMPS [19], для определения межатомного взаимодействия взят потенциал ЕАМ для никеля [20], который для каждого атома имеет вид

$$
E_{i}=F\left(\sum_{j \neq i} \rho\left(r_{i j}\right)\right)+\frac{1}{2} \sum_{j \neq i} \varphi\left(r_{i j}\right),
$$

где $F-$ энергия погружения, являющаяся функцией атомной электронной плотности, $\rho$ - электронная плотность, $\varphi$ - парное потенциальное взаимодействие. Для численного решения уравнений движения используется алгоритм Верлета в скоростной форме с временным шагом $d t=0.05 \mathrm{fs}$. Визуализация модели и анализ дислокационных структур проводились в Ovito [21].

Сначала был произведен нагрев образца до $300 \mathrm{~K}$, для релаксации системы использовался баростат и термостат (NPT-ансамбль частиц). Для расчета деформации применялся NVE-ансамбль. В начале сжатия задавалась сила давления на образец $2.4 \cdot 10^{-10} \mathrm{~N}$, к торцевым поверхностям прикладывалась сила трения, определенная как сила нормального давления в этих областях, умноженная на коэффициент трения. Температура в процессе моделирования варьировалась в пределах 260-300 K, для поддержания температуры использовался термостат Берендсена. Тензор напряжения определяется как

$$
\sigma_{i j}=\frac{\sum_{k}^{N} m_{k} v_{k i} v_{k j}}{V}+\frac{\sum_{k}^{N^{\prime}} r_{k i} f_{k j}}{V},
$$

где $N, N^{\prime}$ - число атомов в системе, $V$ - объем системы, $m_{k}$ - масса $k$-й частицы, $v-$ проекции скорости $k$-й частицы, $r$ - положение $k$-й частицы, $f-$ вектор силы $k$-й частицы. Второй член в формуле (1) вириальный.

\section{2. Эксперимент и моделирование}

В настоящей работе совместно рассмотрены результаты моделирования пластической деформации сжатием монокристаллов никеля и экспериментальные результаты. Подчеркнем еще раз, что размеры образца при моделировании составляли $7.04 \times 7.04 \times 21.12 \mathrm{~nm}$, а при эксперименте $3 \times 3 \times 6 \mathrm{~mm}$, следовательно, описываемые ниже результаты можно отнести к различным масштабным уровням. Благодаря чему можно рассмотреть целостную картину на нескольких масштабах.
Для [001]-монокристаллов с боковыми гранями $\{110\}$ число равнонагруженных систем сдвига с фактором Шмида 0.41 равно восьми (рис. $1, a$ ). Однако изменения объема кристалла при сжатии образца требуют действия определенных систем скольжения в локальных объемах монокристалла. Еще одной кристаллографической особенностью рассматриваемой ориентации является возможность выхода любой из равнонагруженных плоскостей сдвига на свободную боковую поверхность. Благодаря этому сдвиг может протекать в направлении наименьшего внешнего сопротивления, т.е. в сторону свободных боковых поверхностей в отсутствие обратных напряжений от пуансонов испытательной машины. Рассмотрение действия одной из четырех октаэдрических плоскостей в центральном объеме кристалла показывает, что сдвиг по параллельным октаэдрическим плоскостям может беспрепятственно протекать вдоль любого из двух плотноупакованных направлений в сторону верхнего пуансона испытательной машины или нижнего. В верхней и нижней частях образец ограничен пуансонами и, следовательно, сдвиг в этих областях монокристалла осуществляется главным образом в направлении от пуансона. Резюмируя, отметим, что следует ожидать, что сдвиг в плоскости (1111) от нижнего пуансона будет

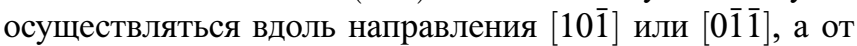
верхнего - [101] или [011]. Основываясь на кристаллографической схеме, можно предположить, что наклонные системы следов сдвига грани (110) сформированы посредством сдвига по плоскостям и, а горизонтальные представляют собой результат сдвига по плоскостям и (рис. 1, b).

Анализируя результаты моделирования, видим, что как горизонтальные (I), так и наклонные системы (II и III) развиваются во всем диапазоне времени моделирования (рис. $1, c-e$ ). Кроме того, можно отметить, что в начальный момент времени дефект упаковки образуется на множестве плоскостей, но с увеличением времени появляется устойчивый рельеф, который представляет собой совокупность наклонных и горизонтальной систем. Это можно связать с тем, что в начале скольжение дислокаций Шокли интенсивно образуется и, встречаясь с противоположными по знаку дислокациями, исчезают. Со временем образуется большое количество пересечение дислокаций, и дальнейшее скольжение становится затрудненным. Процессы, происходящие в кристалле в начале пластической деформации, обусловлены сдвигом в кристалле и нестесненными материальными поворотами [22], а источниками дислокаций в ряде работ называют микромасштабные поверхностные концентраторы напряжений [23], при этом релаксационные процессы реализуются встречными сдвигами.

При увеличении степени деформации образца картина несколько изменяется, что является следствием начала скольжения дислокаций после прорыва барьеров, сформировавшихся вначале нагружения; часть из них опять аннигилирует и возникает другая устойчивая структура. 

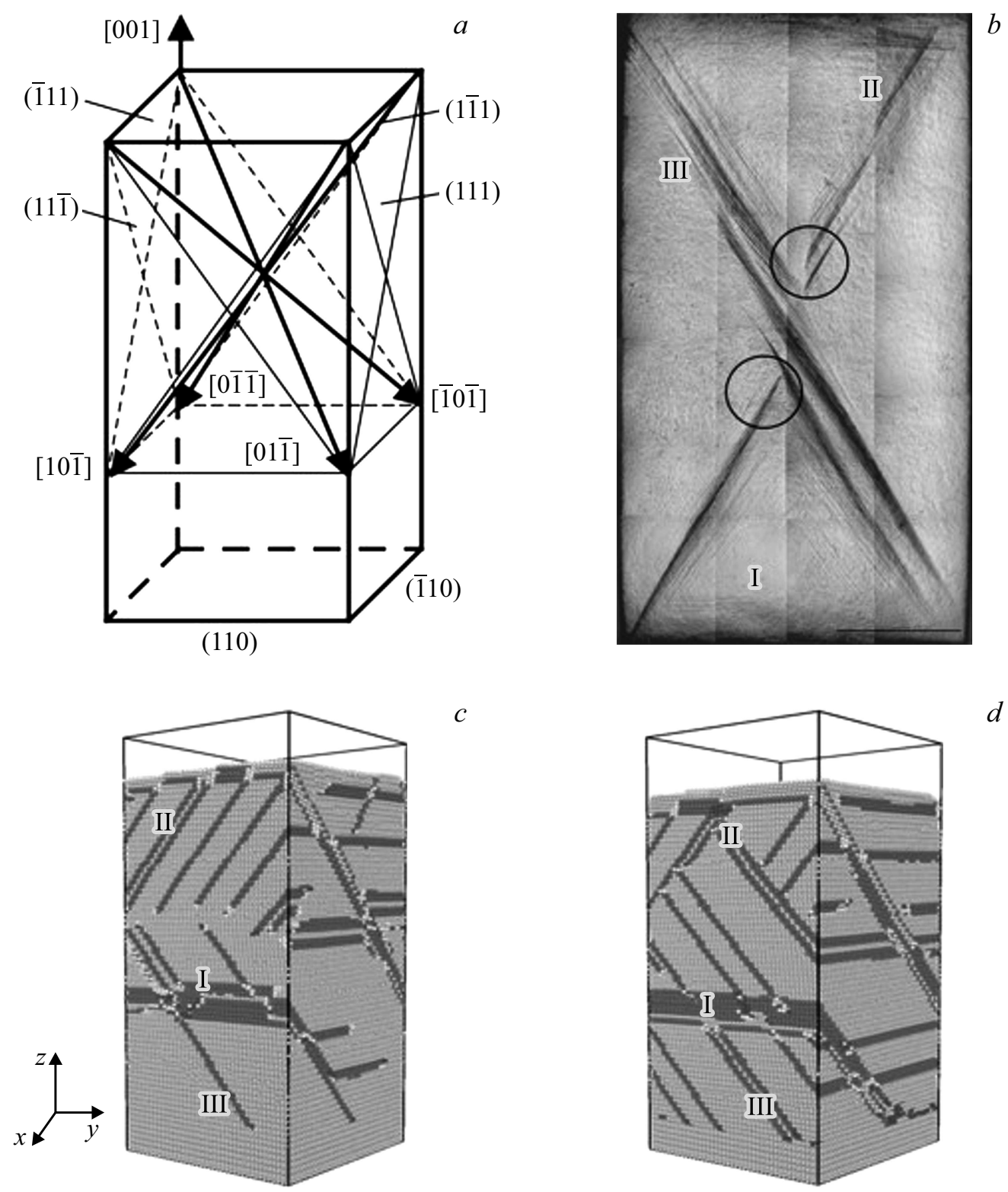

Рис. 1. Кристаллографическая схема [001]-монокристалла с боковыми гранями $\{110\}(a)$; деформационный рельеф на боковой грани (110) при $e=6 \%(b)$; рельеф образца, результаты моделирования при времени $18.35(c)$ и $23.35 \mathrm{ps}(d)$.

Кроме того, можно наблюдать, что действие наклонных систем сдвига происходит поочередно (рис. $1, c-e$ ).

При моделировании на ранней стадии (до $12-14 \mathrm{ps}$ ) было отмечено возникновение сначала ОЦК, а потом и ГПУ-структуры. Их развитие происходило в слоистой структуре вдоль плотноупакованных плоскостей. Учитывая высокую энергию дефекта упаковки никеля, можно полагать, что дислокационное скольжение, развиваясь вдоль плотноупакованных плоскостей, может привести к созданию дислокационной структуры с очень высокой плотностью дефектов укладки в трансформированной ГЦК-фазе. Подобные результаты были получены в работе [24] при рассмотрении прокатки нанокристаллического никеля и в работе [16] при моделировании ударного сжатия монокристаллов никеля с ориентаци- ями осей, сжатых в углах стандартного стереографического треугольника. В работе [25] при моделировании растяжения нанокристаллов меди вдоль оси [001] показано, что за пластическую деформацию ответственно термоупругое мартенситное превращение при котором ГЦК-решетка перестраивается в ГПУ. В работе [16] при моделировании ударной волны в монокристалле никеля также наблюдался переход ГЦК в ОЦК. Причем это характерно для систем, где нагружение идет вдоль оси типа [100]. В настоящей работе с использованием метода МД моделировалась деформация сжатием вдоль направления [001] и наблюдалась ударная волна, следовательно, можно полагать, что наблюдаемая трансформация кристаллической решетки относится к мартенситному превращению. В экспериментальном исследовании [26], 

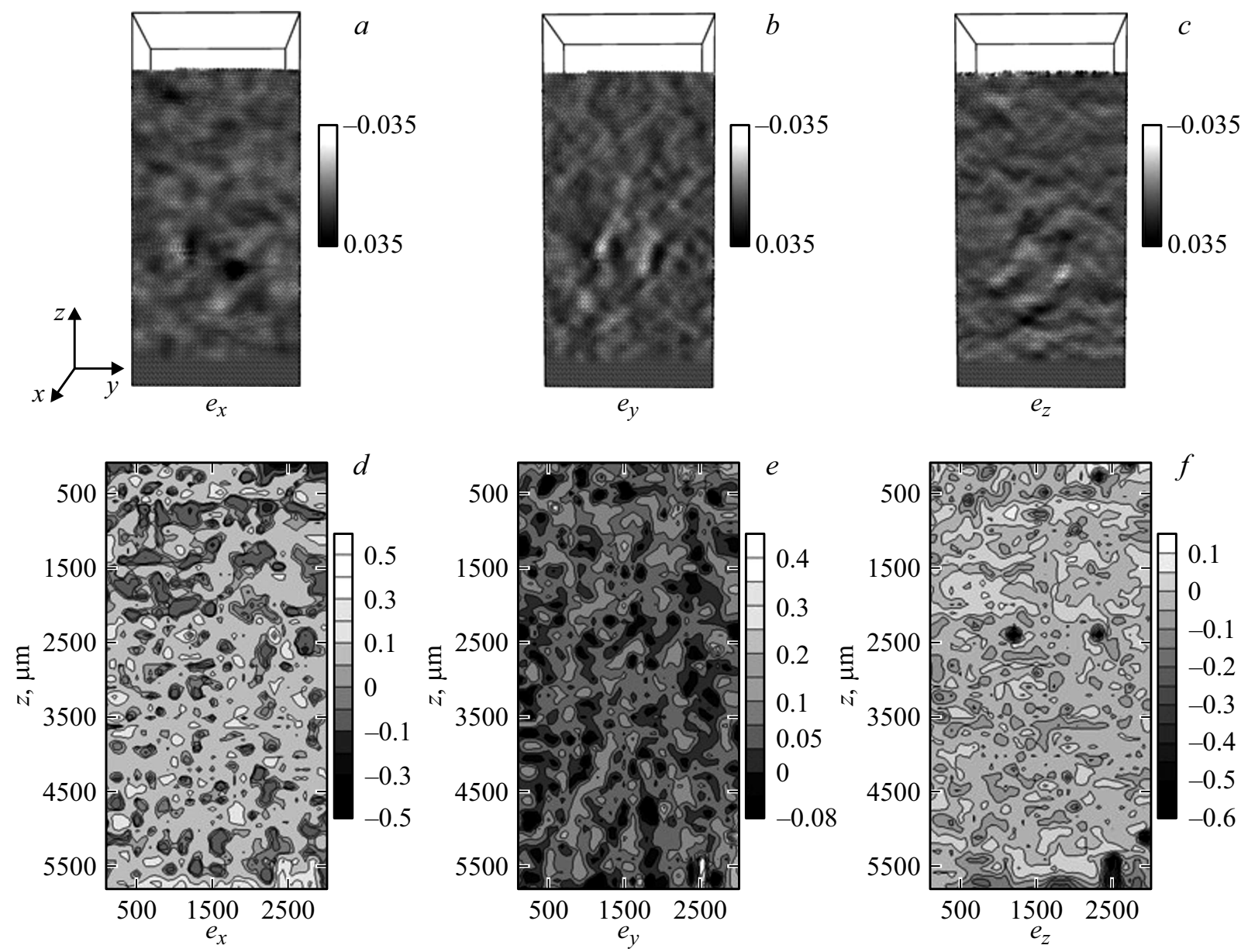

Рис. 2. Карты распределения компонент деформации на боковых гранях $\{110\}$ монокристалла никеля с ориентацией оси сжатия [001]: моделирование при времени $24.85 \mathrm{ps}(a-c)$; эксперимент, $e=6 \%(d-f)$. Компоненты указаны на картах.

где рассмотрено фазовое превращение решетки ГЦК в ОЦК в кристалле никеля при прокатке образца, возникали переходы в другую фазу и сделан вывод, что при большой деформации мартенситное превращение возможно. В нашем исследовании при МД моделировании монокристалл был под большой нагрузкой, что и объясняет фазовый переход ОЦК в ГЦК. Такое же поведение никеля отмечено в работе [27], но в данном случае моделировались не ударные волны, а исследовались нелинейные эффекты при одноосном сжатии и растяжении (кроме молекулярной динамики применялся метод Монте-Карло). Такие переходы можно описать схемой Бейна [28]. В нашей работе при дальнейшем сжатии наблюдается переход из ОЦК в ГПУ решетку. В работе [17] показано, что при моделировании ударных нагрузок вдоль оси типа [100] ОЦК решетка в кристалле железа в основном переходит в ГПУ-решетку.

При натурном эксперименте на макроуровне была получена картина сдвига, представленная на рис. $1, b$. Мы можем наблюдать наклонные системы следов сдвига, идущие от вершин образца (II и III), и горизонтальные следы (I), формирующиеся у торцов кристалла. При этом одна из макрополос явно получает большее развитие. Вероятно, в первоначальный момент времени запускается одна система следов, высокая плотность дислокаций в которой затрудняет развитие второй системы.

Далее рассмотрим распределение компонент деформации. Результаты, полученные моделированием, показаны на рис. 2,a-c. Мы можем наблюдать квазипериодическое распределение локальных областей растяжения/сжатия как на поверхности образца, так и при продвижении вглубь кристалла. Места некоторой локализации деформации на поверхности располагаются вблизи стыка пачек следов (соответствующие области отмечены на рис. $1, b$ окружностями).

В эксперименте общая деформация монокристалла составляет $e=6 \%$, средние значения компонент деформации $-\left\langle e_{x}\right\rangle=0.04,\left\langle e_{y}\right\rangle=0.04,\left\langle e_{z}\right\rangle=-0.07$ (здесь и далее знак „минус“ - это деформация сжатием, „Плюс“ - растяжением). На рис. $2, d-f$ представлены карты распределения компонент $e_{x}, e_{y}, e_{z}$ деформации.

Анализ карт показывает, что деформация распределена по граням достаточно однородно по всем компонентам. Местами некоторой локализации деформации являются приторцевые ребра образца, так как они яв- 

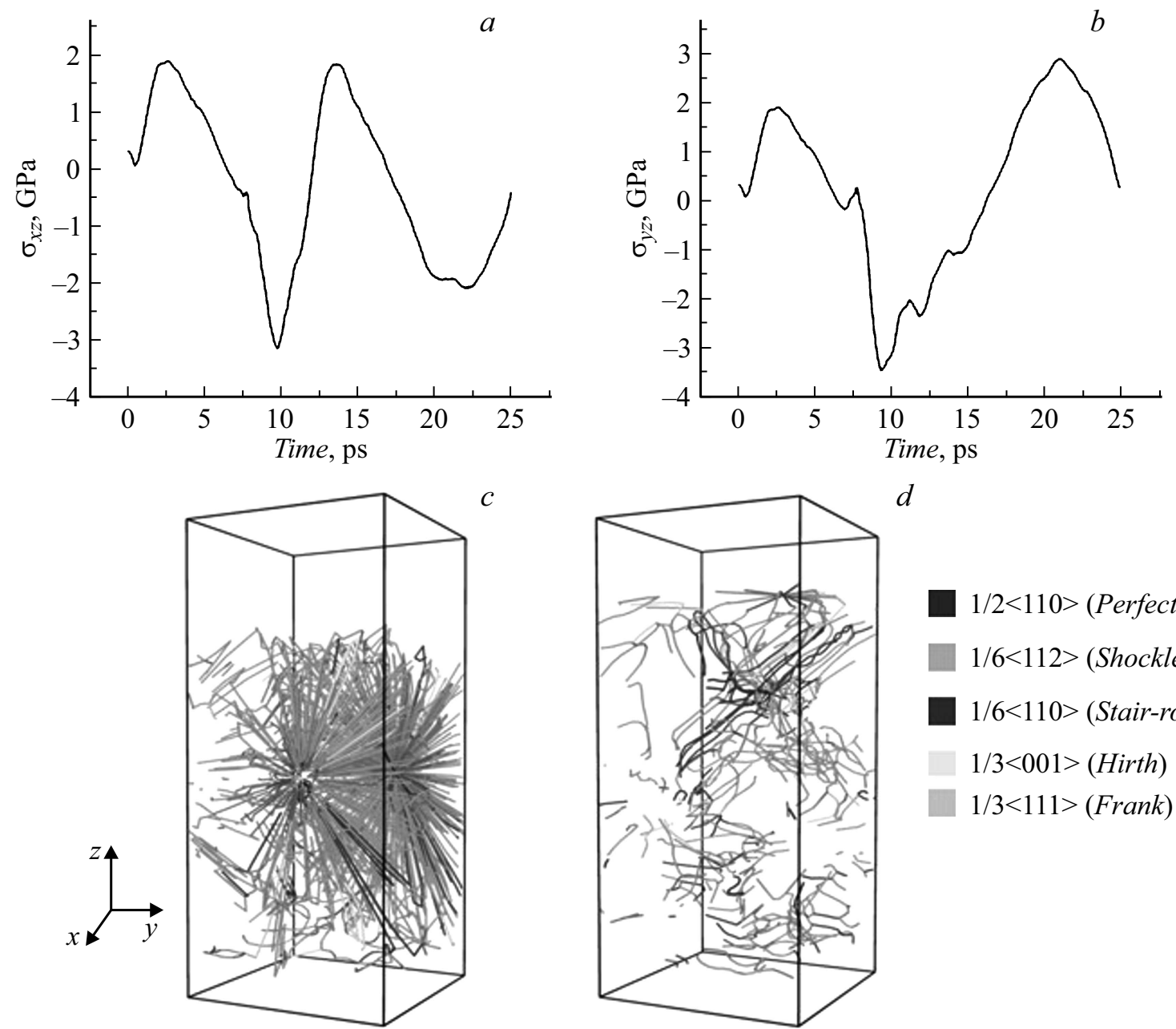

$1 / 2<110>($ Perfect $)$
$1 / 6<112>($ Shockley $)$
$1 / 6<110>($ Stair-rod $)$
$1 / 3<001>($ Hirth $)$
$1 / 3<111>($ Frank $)$

Рис. 3. Зависимость касательных компонент тензора напряжения от времени $\sigma_{x z}(a), \sigma_{y z}(b)$; дислокационная структура в момент времени $7(c)$ и $13 \mathrm{ps}(d)$.

ляются геометрическими концентраторами напряжений. По компоненте ех в верхней приторцевой области реализуется как деформация растяжением $\left(e_{x}=0.15\right)$, так и деформация сжатием $\left(e_{x}=-0.25\right)$, в нижней части грани ех достигает значения 0.25; величины компонент $e_{y}$ и $e_{z}$ в приторцевых областях достигают максимальных значений 0.30 и -0.45 соответственно. Следовательно, также можно говорить о чередовании локальных областей растяжения/сжатия (квазипериодический характер распределения компонент деформации).

Таким образом, и в моделировании, и в эксперименте мы наблюдали равномерное квазипериодическое распределение компонент деформации. Вместе с тем известно, что рельеф, сформировавшийся на поверхности, есть отражение внутренних процессов пластической деформации, произошедших в кристалле [29], поэтому на основе рассмотрения деформационного рельефа можно проанализировать внутренние процессы. В настоящей работе деформационный рельеф был изучен с применением фрактального анализа на основе корреляционной функции „высота-высота“ $H(r)[30,31]$

$$
H(r)=\left\langle\left[Z\left(r^{\prime}\right)-Z\left(r^{\prime}-r\right)\right]^{2}\right\rangle,
$$

где $Z(r)$ - функция высоты поверхности по всем парам точек, отстоящим друг от друга на фиксированное расстояние $r$, скобки указывают на усреднение по всем парам точек.

Далее по графику корреляционной функции $H(r)$, изображенному в логарифмических координатных осях, по наклону начального участка кривой определяется показатель Херста $(H)$, а по проекции линейного участка на ось абсцисс - корреляционная длина $L$. Показатель Херста $H$ можно применить для анализа способности системы к самоорганизации [32].

Расчеты, проведенные для области занятой наклонной системой следов сдвига при деформации $e=6 \%$, дали следующие результаты. На корреляционной длине $L_{1}=1.8 \mu \mathrm{m}$ индекс Херста $H_{1}=0.007$, на $L_{2}=21 \mu \mathrm{m}$ $H_{2}=0.036$, на $L_{3}=67 \mu \mathrm{m} H_{3}=0.285$, на $L_{4}=122 \mu \mathrm{m}$ $H_{4}=0.323$. Корреляционные длины $L_{2}-L_{4}$ по своему масштабу относятся к мезоуровню. 

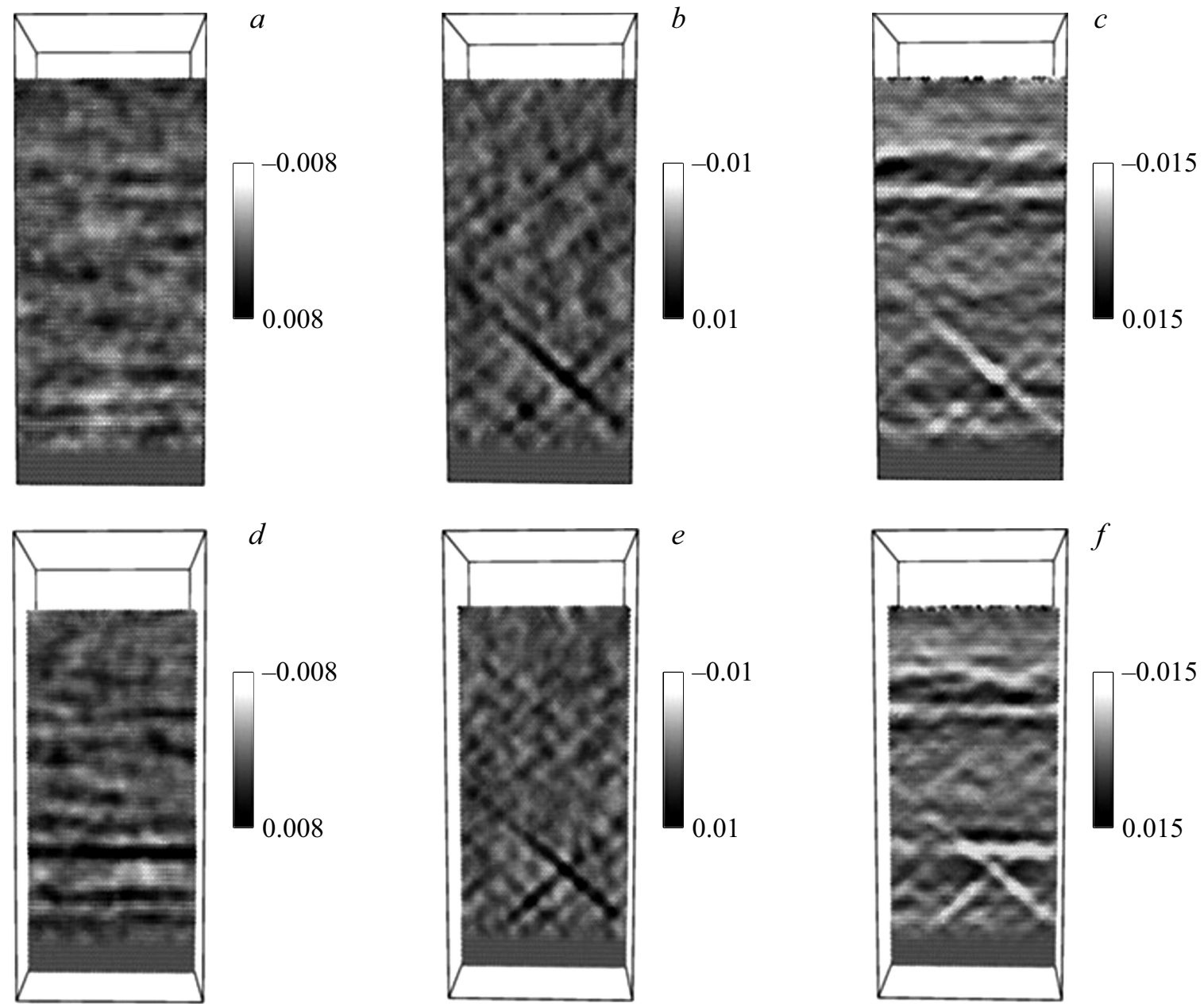

Рис. 4. Распределение компонент деформации в процессе образования дефекта упаковки, время моделирования 6.85 рs; компоненты деформации указаны на картах $(a-c)$ на поверхности образца; срез внутри образца на глубину $3.52 \mathrm{~nm}$ от поверхности $(d-f)$.

Значения показателя Херста меньше 0.5 указывают на то, что система имеет антиперсистентный, эргодический характер, т.е. стремится сохранить среднее значение анализируемого показателя. При этом чем ближе значение $H$ к нулю, тем выше устойчивость системы. Значение $H=0.5$ свидетельствует об отсутствие корреляции [6]. Следовательно, наиболее устойчивой система является на корреляционной длине $L_{1}$, масштаб которой соответствует уровню дислокационной структуры. На поверхности кристалла это отражается в формировании рельефа в виде отдельных следов скольжения (при оптическом увеличении не разрешаются). Результаты моделирования показывают, что на микроуровне происходит чередование периодов интенсивного роста дислокаций и их аннигиляции. На рис. 3 показано поведение касательных напряжений, так как они отражают процессы зарождения и движения дислокаций, то по ним можно отследить поведение дислокационной структуры образца и картину распределения дислокаций в образце (рис. $3, c, d$ ). На рис. $3, a, b$ поведение касательных напряжений схоже до 10 ps. Первый минимум (примерно $1 \mathrm{ps}$ ) можно связать с образованием ударной волны, второй минимум $(7 \mathrm{ps})$, более выраженный на рис. $4, b$, соответствует появлению дефекта упаковки. В это время происходит интенсивный рост дислокаций, на рис. $4, a, b$ это отражается в падении напряжений, с $10-13$ ps роста дислокаций не происходит и значение $\sigma_{x z}$ увеличивается. Известно, что дефекту упаковки сопутствует избыток энергии, поэтому он стремится уменьшить свою площадь [33]. Последовательное рассмотрение карт распределения компонент деформации в процессе формирования дефекта упаковки показывает наличие мест локализации компонент деформации вдоль плотноупакованных октаэдрических плоскостей как по наклонным, так и по горизонтальной системе (рис. 4). Вместе с тем при сравнение картины распределения компонент на грани кристалла и внутри показывает чередование компонент растяжения/сжатия в направлении от поверхности вглубь, таким образом, благодаря кооперативным процессам в дислокационной структуре происходит релаксация напряжений в кристалле. 
Следовательно, как в моделировании, так и в практическом эксперименте мы наблюдаем способность кристалла к самоорганизации пластической деформации с целью сохранения своей целостности (живучесть системы).

На следующих масштабных уровнях (корреляционные длины $\left.L_{2}-L_{4}\right)$, которые соответствуют размеру пачки плоскостей сдвига (состоит из множества параллельных следов, которые в свою очередь состоят из группы следов скольжения), также можно видеть тенденцию к сохранению квазипериодического характера формирования профиля поверхности, хотя и выраженное в меньшей степени (значения индекса Херста не достигает 0.5$)$.

\section{Заключение}

Проведенное в работе совместное рассмотрение результатов математического моделирования и экспериментальных исследований показало хорошую согласованность результатов и позволило показать целостную картину протекания пластической деформации на разных масштабных уровнях.

Моделирование показало, что для такого рода деформации (одноосное сжатие, малый размер образца, большая нагрузка) характерны фазовые переходы кристаллической решетки: сначала ГЦК-ОЦК, затем ОЦК-ГПУ. Перестройка кристаллической решетки может быть одним из вкладов в самоорганизацию пластической деформации кристалла. Кроме того, на атомном уровне отмечена склонность к образованию устойчивых дислокационных структур - в начале сжатия дислокации образуются в большом количестве на многих системах скольжения, в дальнейшем образуется устойчивая крестообразная структура из пересечения дислокаций, которая остается на протяжении всего моделирования.

Установлено, что способность к самоорганизации пластической деформации с целью сохранения своей целостности присуща кристаллу на всех масштабных уровнях и наиболее сильно проявляется на микроуровне. Данная закономерность подтверждена как в моделировании, так и в эксперименте.

Благодаря фрактальному анализу определены значения показателя Херста и корреляционные длины для [001]-монокристалла с боковыми гранями $\{110\}$. Разница в величине показателя Херста на микро- и мезоуровне указывает на различие в механизмах самоорганизации пластической деформации. На микроуровне механизмом является поочередное зарождение и аннигиляция дислокаций. На этом уровне способность к сохранению целостности самая высокая. На мезоуровне самоорганизация осуществляется за счет коррелированного сдвига в параллельных плоскостях скольжения. Благодаря указанным процессам мы можем наблюдать равномерное квазипериодическое распределение компонент деформа- ции по грани, что способствует сохранению живучести кристалла.

Экспериментальны часть исследования выполнена при финансовой поддержке РФФИ в рамках научного проекта № 16-32-60007 мол_а_дк.

Молекулярно-динамическое моделирование выполнено при поддержке Министерства образования и науки Российской Федерации в рамках государственного задания 3.2510.2017/ПЧ.

Авторы выражают благодарность профессору, д.ф.-м.н. Д.В. Лычагину за поддержку работы и к.ф.-м.н. В.С. Красникову за помощь в моделировании.

\section{Список литературы}

[1] Kahloun C., Monnet G., Queyreau S., Le L.T., Franciosi P. // Int. J. Plast. 2016. Vol. 84. N 6. P. 277-298.

DOI: $10.1016 /$ j.ijplas.2016.06.002.

[2] Cai M., Langford S.C., Thomas Dickinson J. // Acta Mater. 2008. Vol. 56. N 20. P. 5938-5945.

DOI: 10.1016/j.actamat.2008.08.015.

[3] Kramer D.E., Savage M.F., Levine L.E. // Acta Mater. 2005. Vol. 53. N 17. P. 4655- 4664.

DOI: $10.1016 /$ j.actamat.2005.06.019

[4] Mecif A., Bacroix B., Franciosi P. // Acta Mater. 1997. Vol. 45. N 1. P. 371-381. http://dx.doi.org/10.1016/S13596454(96)00094-8.

[5] Лычагин Д.В., Алферова Е.А., Старенченко В.А. // Физическая мезомеханика. 2010. Т. 13. Вып. 3. С. 75-88. [Lychagin D.V., Alfyorova E.A., Starenchenko V.A. // Phys. Mesomech. 2011. Vol. 14. N 1-2. P. 66-78.]

[6] Ляпунова Е.А., Петрова А.Н., Бродова И.Г., Наймарк О.Б., Соковиков М.А., Чудинов В.В., Уваров С.В. // Физическая мезомеханика. 2012. Т. 2. Вып. 15. С. 61-67.

[7] Alfyorova E.A., Lychagin D.V. // Mech. Mater. 2018. Vol. 117. P. 202-213. DOI: 10.1016/j.mechmat.2017.11.011

[8] Alfyorova E.A., Lychagin D.V. // Lett. Mater. 2017. Vol. 7. N 2. DOI: $10.22226 / 2410-3535-2017-2-155-159$

[9] Girardin G., Huvier C., Delafosse D., Feaugas X. // Acta Mater. 2015. Vol. 91. P. 141-151. DOI: $10.1016 /$ j.actamat.2015.03.016

[10] Малыгин Г.А. // УФН. 1999. Т. 169. № 9. С. 979-1010.

[11] Панин В.Е., Егорушкин В.Е., Панин А.В. // Физическая мезомеханика. 2006. Т. 8. Вып. 3. С. 9-22.

[12] Малыгин Г.А. // ФТТ. 2007. Т. 49. Вып. 8. С. 1392-1397.

[13] Красников В.С., Куксин А.Ю., Майер А.Е., Янилкин А.В. // ФТТ. 2010. Т. 52. Вып. 7. С. 1295-1304.

[14] Krasnikov V.S., Mayer A.E., Yalovets A.P. // Int. J. Plast. 2011. Vol. 27. N 5. P. 1294-1308.

DOI: $10.1016 /$ j.jplas.2011.02.008

[15] Komanduria R., Chandrasekaran N., Raff L.M. // Int. J. Mech. Sci. 2001. Vol. 43. P. 2237-2260.

[16] Chen Y., Zhou1 L., He W., Sun Y., Li Y., Jiao Y., Luo S. // Eur. Phys. J. B. 2017. Vol. 90. N 16. DOI: 10.1140/epjb/e201670388-7

[17] Wang K., Xiao S., Deng H., Zhu W., Hu W. // Int. J. Plasticity. 2014. Vol. 59. P. 180-198. DOI: 10.1016/j.ijplas.2014.03.007

[18] Кукса Л.В., Ковальчук Б.И., Лебедев А.А. и др. // Проблемы прочности. 1976. № 3. С. 55-59. 
[19] Plimpton S. // J. Comp. Phys. 1995. Vol. 117. P. 1-19.

DOI: $10.1006 / \mathrm{jcph} .1995 .1039$

[20] Mishin Y., Farkas D., Mehl M.J., Papaconstantopoulos D.A. //

Phys. Rev. B 1999. Vol. 59. P. 3393. DOI: $10.1103 /$ PhysRevB.59.3393

[21] Stukowski A. // Model. Simul. Mater. Sci. Eng. 2010. Vol. 18. P. 015012.

[22] Панин В.Е., Панин Л.Е. // Физическая мезомеханика. 2004. Т. 4. Вып. 7. Р. 5-23.

[23] Конева Н.A. // Соросовский образовательный журнал. 1996. № 6. P. 99-107.

[24] Zhang X.Y., Wu X.L., Liu Q., Zuo R.L., Zhu A.W., Jiang P., Wei Q.M. // Appl. Phys. Lett. 2008. Vol. 93. P. 031901. DOI: $10.1063 / 1.3062849$

[25] Золотых Т.А., Косилов В.В., Ожерельев В.В. // Компьютерные исследования и моделирование. 2013. Т. 5. № 2. C. 225-230.

[26] Zhang X.Y., Wu X.L., Liu Q., Zuo R.L., Zhu A.W., Jiang P., Wei Q.M. // Appl. Phys. Lett. 2008. Vol. 93. P. 031901. DOI: $10.1063 / 1.2953545$

[27] Wen Y., Wu S., Zhang J., Zhu Z. // Solid State Commun. 2008. Vol. 146. P. 253-257.

[28] Гуляев А.П. Металловедение. Учебник для вузов. 6-е изд. М.: Металлургия, 1986. 544 с.

[29] Хирт Дж., Лоте И. Теория дислокаций. М.: Атомиздат, 1972. $600 \mathrm{c}$

[30] Yang H.-N., Zhao Y.-P., Chan A., Lu T.-M., Wang G.C. // Phys. Rev. B. 1997. Vol. 56. N 7. P. 4224-4232. DOI: $10.1103 /$ PhysRevB.56.4224

[31] Pelliccione M., Lu T.-M. Evolution of Thin Film Morphology. Modeling and Simulations. NY.: Springer, 2008. 206 p.

[32] Аптуков В.Н., Митин В.Ю., Скачков А.П. // Вестник Пермского ун-та. Математика. Механика. Информатика. 2010. Т. 4. Вып. 4. С. 30-33.

[33] Новиков И.И. Дефекты кристаллического строения металлов. Уч. пособ. для вуза. М.: Металлургия, 1975. 208 с. 